AperTO - Archivio Istituzionale Open Access dell'Università di Torino

\title{
Stranieri a tavola? Ridefinire i confini identitari attraverso le narrazioni alimentari
}

\section{This is the author's manuscript}

Original Citation:

Availability:

This version is available http://hdl.handle.net/2318/1806807

since 2022-03-28T07:04:42Z

Published version:

DOI:10.3280/MM2021-002008

Terms of use:

Open Access

Anyone can freely access the full text of works made available as "Open Access". Works made available under a Creative Commons license can be used according to the terms and conditions of said license. Use of all other works requires consent of the right holder (author or publisher) if not exempted from copyright protection by the applicable law. 


\title{
Stranieri a tavola? Ridefinire i confini identitari attraverso le narrazioni alimentari
}

\author{
di AliceScavarda*, Raffaella Ferrero Camoletto ${ }^{* *}$ \\ e Vulca Fidolini ${ }^{* * *}$
}

Riassunto: Attraverso l'analisi di tre casi di studio che utilizzano tre approcci qualitativi diversi (l'intervista, la photovoice, l'etnografia virtuale), il presente articolo mostra come l'alimentazione nell'esperienza migratoria possa fungere da spazio, sociale e virtuale, reale e immaginario, per rivendicare dei confini simbolici che possono essere costruiti in modo nostalgico o riaffermati in quanto tratto distintivo che differenzia chi migra dall'autoctono. Allo stesso tempo, il cibo può operare come terreno di incontro e di ibridazione di tradizioni diverse, disvelando versanti talvolta insoliti o nascosti dei processi migratori.

Parole-chiave: cibo; migrazioni; stigma; interviste; photovoice; etnografia virtuale.

Strangers at the table? Redefining identity boundaries through food narratives

Abstract: Through the analysis of three case studies adopting three different qualitative methods, namely semi-structured interviews, Photovoice and digital ethnography, the paper analyses how food within migration represents a space, either real or virtual, either real or imagined, to claim a cultural authenticity. The latter can be constructed both with nostalgia and reasserted as a distinctive trait, able to differentiate who migrates from native population. In parallel, food can allow a hybridization of different traditions, unravelling hidden and unusual aspects of migration processes.Keywords: food; migrations; stigma; interviews; photovoice; virtual ethnography.

Ricevuto: 26 Novembre 2020 Accettato: 18 Marzo 2021

* Alice Scavarda, Università degli Studi di Torino (Italia); alice.scavarda@unito.it.

**. Raffaella Ferrero Camoletto, Università degli Studi di Torino (Italia); raffaella.ferrerocamoletto@unito.it

***.Vulca Fidolini, Université de Lorraine (Francia); vulca.fidolini@univ-lorraine.fr.

Mondi Migranti, (xxxx-xxxx, ISSNe xxxx-xxxx), 2/2021

Commentato [A1]: Gentilissime autrici vi chiedo di verificare queste date (le ho copiate da ojs, a me sembrano ok...) e i vostri riferimenti presenti in nota a fondo pag. Grazie 


\section{Introduzione}

Lo studio del cibo nelle migrazioni è centrale oggi nel dibattito delle scienze sociali, e in particolar modo nella sociologia e nell'antropologia. Se è vero che gli studi anglosassoni e americani hanno aperto la strada a tali approcci ormai da tempo (Gabaccia, 1998; Ray, 2004), negli ultimi anni un nuovo impulso arriva soprattutto dall'Europa continentale (Janowski, 2012). La letteratura evidenzia quanto la migrazione implichi la necessità di ripensare il rapporto col cibo (d'origine) e con nuove pratiche e abitudini alimentari (Crenn, 2020). Quando si parla di alimentazione, infatti, non si intende solo il cibo, ma anche, e soprattutto, rappresentazioni sociali, norme e costumi (Fischler, 1992). Certo, il cibo è innanzitutto nutrimento e bisogno fisiologico, ma, da un punto di vista sociologico, è anche strumento culturale, veicolo di memoria, emozioni, relazioni che accompagnano e modellano i percorsi dei migranti, permettendoci di scoprirne versanti talvolta nascosti (Terragni e Roos, 2018). Ripensare le proprie abitudini alimentari e confrontarsi con nuovi codici culinari può rivelarsi complicato per chi migra; trasformare il cibo in ripiego identitario intra-comunitario per non aprirsi all'altro è una delle possibili conseguenze, così come il rischio di subire stigmatizzazioni per preferenze culinarie troppo lontane da quelle di chi accoglie; o ancora, adattarsi al "cibo dell'altro" in una logica assimilazionista, per ottenere riconoscimento attraverso le proprie pratiche di consumo; o ancora, ibridare il "proprio" cibo con quello della società di accoglienza, per riuscire a passare per un membro del gruppo maggioritario. Questo uso del cibo può costituire una perfetta applicazione di quel processo denominato boundarymaking, secondo cui l'identità non costituisce una caratteristica pre-esistente e ascritta ad un gruppo, ma è bensì un prodotto di un processo di continua costruzione e ridefinizione di confini relazionali tra gruppi sociali (Lamont et al., 2001).

Nel presente testo ci interesseremo a come il cibo "racconti le migrazioni" e a come lo si racconti "nella migrazione" (Raman, 2011), e a come il cibo permetta di (ri)costruire i confini simbolici. L'attenzione sarà concentrata su tre casi studio ${ }^{1}$ che metteranno in risalto le peculiarità di tre differenti esperienze migratorie e di tre approcci qualitativi diversi: dall'intervista, alla photovoice, all'etnografia virtuale. I fenomeni migratori che analizzeremo si di-

1. Il caso studio n. 1, oggetto del par. 2, è stato analizzato da Vulca Fidolini, il caso studio n. 2, oggetto del par. 3, è stato analizzato da Alice Scavarda e il caso studio n. 3, oggetto del par. 4, da Raffaella Ferrero Camoletto. I parr. Introduzione, par. 1 e Conclusioni sono il frutto del lavoro comune degli autori.
Commentato [A2]: Ho eliminato il numero del paragrafo perché introduzione e conclusione per gli articoli di Mondi Migranti non hanno numero di paragrafo e di conseguenza ho rinumerato i par. successivi. Vi chiederei di verificare la correttezza della nota a fondo paginperchè anche lì ho modificato i numeri dei paragrafi. grazie 
stinguono principalmente per le motivazioni che li ispirano, per gli spostamenti che implicano (interni ai confini nazionali o internazionali) ${ }^{2}$. Un primo gruppo di migranti cui ci interesseremo è rappresentato da giovani maschi marocchini arrivati in Italia e in Francia per motivi di lavoro o di studio; un secondo gruppo è composto da studenti e studentesse e lavoratori e lavoratrici giunti in Italia da differenti paesi, individualmente o tramite ricongiungimenti familiari; un terzo gruppo è costituito invece da giovani ragazze e ragazzi del Sud Italia che hanno raggiunto il Nord per ragioni di studio. Quest'ultimo gruppo ci permetterà di arricchire il nostro sguardo sul cibo nelle migrazioni. Le popolazioni migranti interne, infatti, godono solitamente degli stessi diritti di quelle autoctone, e condividono con chi accoglie la stessa lingua e uno stesso universo culturale di riferimento che, seppur vasto, è riconducibile a forti tratti comuni (la confessione religiosa per esempio). Il tema del cibo diventerà una sorta di (pre)testo interpretativo per leggere i processi dialettici di boundary-making che attraversano i fenomeni migratori presi in esame e che operano sul piano del rapporto tra le generazioni, all'interno delle asimmetrie di genere, $\mathrm{o}$ ancora nel confronto tra popolazioni autoctone $\mathrm{e}$ straniere. La dimensione rituale del cibo consentirà di cogliere il senso di queste dialettiche e delle pratiche di spaesamento e ri-appaesamento che accompagnano i fenomeni migratori (Cottino, 2020). L'alimentazione sarà l'oggetto analitico tramite cui sondare le molteplici negoziazioni di confini, individuali e collettivi, messe in atto dagli intervistati e dalle intervistate per comporre e ricomporre i loro percorsi identitari e migratori.

\section{Narrare il cibo per parole e immagini}

Il dibattito sulle tecniche d'indagine più appropriate per lo studio delle pratiche alimentari - specie nel loro dialogo con le dinamiche migratorie non è nuovo (Crenn, 2020). Pur riconoscendo le potenzialità delle ricerche quantitative in termini di generalizzazione dei risultati acquisiti, la letteratura ne ha messo in evidenza numerosi limiti (Poulain, 2008): soprattutto quando l'obiettivo, come nel nostro caso, è quello di indagare i processi di costruzione dei significati e dei confini simbolici messi in atto attraverso le pratiche alimentari nei percorsi migratori, il ricorso a tecniche di ricerca qualitative appare una opzione euristicamente più feconda.

2. In una logica intersezionale potrebbero certamente essere individuate altre dimensioni che differenziano internamente i flussi migratori (in termini di genere, o di età, $o$ ancora di classe sociale). Nella nostra analisi abbiamo specificato quando tali dimensioni ci sembravano giocare un ruolo rilevante a livello interpretativo. 
Nel presente testo abbiamo scelto di focalizzare l'attenzione su tre tecniche qualitative: le interviste in profondità accompagnate dall'osservazione etnografica; i metodi visuali, con particolare attenzione alla photovoice; e i metodi digitali, con specifico riferimento all'etnografia virtuale. In letteratura si trovano numerosi esempi dell'impiego di queste tecniche di ricerca. Bojorquez, Rentería e Unikel (2014) hanno investigato le migrazioni interne in Messico, dalle zone rurali a quelle urbanizzate tramite interviste semidirettive e osservazione: lo studio rende conto di come la transizione da una dieta ricca di vegetali e cereali verso una più incentrata su alimenti proteici e processati industrialmente si accompagni alla rivendicazione di un affrancamento dalle tradizioni alimentari familiari, tramite l'adozione di regimi personalizzati e aperti alla sperimentazione di nuovi prodotti. I metodi visuali e digitali, dal canto loro, rispondono alla necessità contemporanea di studiare il vasto mondo dei social media in quanto arene di condivisione di rappresentazioni culturali disparate relative all'alimentazione (Taylor e Keating 2018). La spettacolarizzazione del cibo innesca un processo di feticizzazione capace di creare specifici foodscapes (Stagi, 2016; Middha, 2018). Due contributi ne esemplificano la fecondità euristica nello studio delle migrazioni. La ricerca di Soriano-Ayala, Cala e Ruiz-Salvador (2020) utilizza le immagini (foto e video) per esplorare le percezioni degli stili di vita "sani" tra gli e le adolescenti spagnoli/e originari/e della Romania e del Marocco. I metodi visuali permettono agli autori di rivelare come i giovani di origini rumene adottino pratiche mimetiche che li avvicinano a un modello di dieta occidentale-mediterranea, mentre i giovani marocchini enfatizzino le origini con riferimenti al valore simbolico del "loro cibo tradizionale" e tramite il riconoscimento delle madri in quanto depositarie dell'originalità delle ricette. La ricerca condotta da Lin, Pang e Liao (2020), infine, ricorre a una etnografia online e offline per esplorare la produzione domestica di alimenti in un campione di donne taiwanesi residenti in Belgio. L'attenzione è concentrata su come le donne migranti facciano del cibo uno strumento per negoziare la propria identità all'estero.

Gli approcci qualitativi si rivelano uno strumento euristico per studiare le pratiche alimentari in quanto terreno di negoziazione, nel contesto della migrazione, dei confini simbolici che definiscono le proprie origini geografiche e le proprie identità culturali. Tali tecniche permettono non solo di porre l'attenzione sui significati plurali e cangianti che l'alimentazione può assumere nelle migrazioni, ma anche di confrontare tra loro narrazioni del cibo fatte di parole e immagini raccolte in modo differente: tramite resoconti di intervista; fotografie autoprodotte; video, foto, meme e testi postati sui social media. L'approccio metodologico si avvicina a quello dei casi massimamente dissimili (Cardano, 2020): se in condizioni molto differenti tra loro si ritrovano 
dinamiche simili, ciò significa che si tratta di processi molto rilevanti nel fenomeno in studio. Aver raggiunto conclusioni simili partendo da tre ricerche che hanno preso in esame aspetti diversi del fenomeno migratorio, attraverso una varietà di tecniche di ricerca, rafforza la solidità dei risultati ottenuti.

\section{Purezza vs pericolo: l'etnografia e il nesso tra cibo e sessualità tra i migranti musulmani}

Il primo studio riguarda una popolazione di giovani maschi marocchini, musulmani (più di sessanta in totale, originari del Marocco urbano e rurale, e con origini sociali differenziate), emigrati in Italia e in Francia. La ricerca ha esplorato come le esperienze della sessualità e dell'identificazione di genere - maschili, in particolare - operassero all'interno di un percorso migratorio situato durante la giovinezza ${ }^{3}$.

Pur non trattandosi di una ricerca mirata allo studio delle pratiche alimentari, il cibo è divenuto uno dei temi ricorrenti nelle interviste semi-direttive e nelle osservazioni etnografiche realizzate (Fidolini, 2016; Fidolini, 2019). Durante la ricerca di campo, gli intervistati hanno più volte ricordato quanto fosse per loro più "naturale" rispettare le prescrizioni religiose in materia alimentare rispetto a quelle sessuali, riconoscendo in questo tratto una specificità "tipica dei marocchini e dei musulmani" (Mehdi, 27 anni, Pistoia), non rintracciabile in nessun'altra popolazione europea. "Non avete la cultura del Ramadan nella vostra testa!" asserisce, perentorio, Hamid (27 anni, Mulhouse). Secondo gli intervistati, rinunciare alle relazioni sessuali preconiugali, illecite secondo l'Islam, impedirebbe di sperimentare una sessualità libera prima del matrimonio, e di affermare un modello di maschilità, eterosessuale, debordante e virile. In merito al cibo, invece, sembrano disegnarsi confini più netti.

Ne ho fatti di peccati: ogni volta che vado con una è haram (l'atto illecito, in arabo), non arriverò vergine al matrimonio, ho mentito, ho saltato le preghiere. Ma una cosa non l'ho mai fatta e per me su questo non si discute: non ho mai mangiato maiale (Houcine, 25 anni, operaio, Firenze).

La retorica degli intervistati è interessante perché sembra riconoscere nell'illecito legato al cibo un ostacolo all'espressione di una maschilità forte

3. I giovani sono arrivati in Europa e sono stati intervistati (tra il 2012 e il 2015) quando avevano tra i 18 e i 30 anni di età. 
e dirompente, mentre la letteratura su alimentazione e genere ha mostrato quanto la messa in scena della voracità contribuisca a produrre modelli maschili dominanti, virili e predatori (Fidolini, 2016; Fidolini, 2019).

In tal senso, affrontando il tema della relazione tra corpo puro e impuro attraverso l'alimentazione, Mary Douglas (1973) spiega quanto la mancata osservanza delle prescrizioni religiose sia un terreno privilegiato per creare "disordine" nell'essere umano. Perturbare tale equilibrio richiede il ricorso a pratiche di purificazione che riparino l'ordine e la purezza violati, come esemplifica la vicenda di Taofik, ventiseienne, originario di Casablanca e residente dall'età di 14 anni nella periferia di Strasburgo. Nonostante una relazione di coppia stabile con una ragazza francese non musulmana, nel corso dell'intervista non lesina particolari riguardo le sue scappatelle con partner occasionali. Una sensazione di "disgusto" e "sporcizia" riguardo il proprio corpo accompagnava i rientri a casa ogni volta che si trovava a tradire la compagna, finché un episodio particolare l'ha condotto a rivedere il suo comportamento.

Ero con questa tipa insomma, avevo bevuto, sesso, a un certo punto mi viene fame. Mi dice: “Ti faccio le alette di pollo!", le butta nel microonde, mi dice 'fidati sono halal' ${ }^{4}$ Perché, cioè, vado a spassarmela così, tradisco la mia compagna con cui non sono neanche sposato, bevo, sono nell' haram totale, ma mi preoccupo comunque di mangiare halal! (Taofik, 26 anni, originario di Casablanca e residente dall'età di 14 anni nella periferia di Strasburgo).

In una condizione di completa trasgressione dei dettami religiosi, la riflessione di Taofik sul suo vissuto lo porta ad interpretare l'illecito legato al peccato alimentare in quanto punto di riferimento dal quale ripartire.

Le dico: "Ma sei sicura, le alette?" Insomma alla fine le ho mangiate, erano troppo buone $[\ldots]$ Ho cercato quelle alette nei supermercati per giorni e quando le ho trovate, ho scoperto che non erano affatto halal. Non c'era niente di halal in quella serata, neanche le alette! (Taofik, 26 anni, originario di Casablanca e residente dall'età di 14 anni nella periferia di Strasburgo).

In seguito a questa esperienza Taofik racconta che aver varcato il limite dell'alimentazione prescritta dall'islam l'ha spinto a riflettere. "Non so più quante docce di purificazione abbia fatto nei giorni seguenti" descrivendo la sensazione di sporcizia spirituale che l'attanagliava dopo aver commesso quella violazione, che Taofik riconosceva più grave del peccato sessuale: "Se

4. Cibo "lecito", preparato rispettando i crismi della macellazione della carne secondo i dettami religiosi musulmani. 
hai voglia di sesso, siamo uomini, è dura resistere. Ma per mangiare, hai mille possibilità: potevo mangiare un'altra cosa". Nel racconto di Taofik prende forma un vero e proprio distinguo sul piano della morale e dei valori messi in gioco (Fassin, 2009) per giudicare ciò che può essere giustificato (il sesso fuori dal matrimonio) e ciò che non può esserlo (il consumo di cibo haram). Così, nel tentativo di giustificare un'attitudine maschile dirompente in materia di sessualità, l'intervistato utilizza il potere auto-riflessivo del racconto per giudicare la sua condotta fino ad affermare che quelle alette di pollo sono state "il culmine. Ero andato oltre. Da allora non ho più fatto stupidaggini, la sera resto a casa, tranquillo". L'identità di migrante finisce per essere rivendicata fino a tentare il ribaltamento del rapporto di subalternità col paese che l'ha accolto: "Da quando sono qui ne ho presi di vizi [...]. Io non voglio diventare come fate qui [in Europa], voi ve ne fregate della religione. Per questo ho deciso di mettere la testa a posto dopo quella volta".

\section{Tra nostalgia e ibridazioni culturali: descrivere la propria identità culinaria con la photovoice}

Indagare le modalità d'espressione della propria appartenenza culturale attraverso il cibo era l'obiettivo della seconda indagine qualitativa, realizzata tra Novembre 2017 e Marzo 2018 e basata sulla photovoice, una tecnica visuale tramite cui si studia la percezione soggettiva di immagini con contenuti prodotti dai partecipanti alla ricerca (Harper, 2012), particolarmente utile a rappresentare i significati legati all'alimentazione (Ferrero Camoletto e Scavarda, 2020). Lo studio, supervisionato dall'autrice, ha sollecitato la produzione di fotografie sul rapporto con il cibo da parte di 8 studenti universitari e 7 lavoratori, tra i 17 e i 43 anni, 9 maschi e 6 femmine, giunti a Torino, nel Nord-Italia, da soli o con la famiglia, da diversi paesi europei ed extraeuropei (Romania, Albania, Siria, Brasile, Egitto, Somalia, Portogallo, Costa d'Avorio, Marocco, Perù, Israele, Bolivia, Cina). I partecipanti dovevano produrre immagini raffiguranti il ruolo svolto dal cibo nella vita quotidiana (consolazione, tentazione, piacere, preoccupazione, socialità), inviarle via Whatsapp ai ricercatori e descriverne $\mathrm{i}$ significati nel corso di interviste registrate e poi trascritte.

Nei racconti visuali si possono cogliere diverse strategie di composizione e ricomposizione della cultura di origine attraverso il confronto con quella di arrivo. La nostra attenzione si è concentrata sulle logiche di riappropriazione del vissuto tramite il meccanismo della nostalgia (Librandi, 2005): un sentimento prevalente tra le persone intervistate, specie tra coloro che hanno una storia di migrazione recente e individuale, che diventa strumento critico per 
rielaborare il passato e delineare possibili traiettorie di vita future (Teti, 2020). Come nel caso di una studentessa cinese di 22 anni, proveniente dalla provincia dello Hubei, in Italia da due anni, che attraverso l'immagine di un arrosto di carne con spezie servito con la tipica salsa "plum sauce" mostra una cena con parenti in occasione dell'ultimo suo viaggio di ritorno in Cina: "[Io e miei parenti] festeggiavamo il fatto di essere tutti riuniti e quelle occasioni sono per me cosi ricche di risate ed emozioni che colmano la mancanza per un intero anno". Il cibo appare qui come elemento materiale e simbolico che mantiene vivo il legame con la propria famiglia lontana e con il contesto d'origine. Per altri intervistati, invece, la cui migrazione ha origini più lontane nel tempo ed è spesso stata intrapresa con i genitori, la commistione di pratiche alimentari avviene all'interno dei piatti o dei pasti raffigurati in una singola immagine. Per esempio, in quella che ritrae una tavola imbandita, nella quale compare un piatto della tradizione peruviana, il pato con frejoles (anatra con yuca e fagioli), consumato assieme ad un prodotto tipico italiano, il salame. La tavola imbandita in cui si mescolano la cucina peruviana e italiana produce un'ibridazione delle culture che intende abbattere le distinzioni tra "noi" e "loro". Nel descrivere l'immagine, l'intervistato, un ventitreenne giunto in Italia a cinque anni con la famiglia, allude a un multiculturalismo del quotidiano che, tramite il cibo, fa della differenza un processo di cambiamento e continua integrazione (Colombo e Semi, 2007): "male non fa mischiare le culture, anche nel cibo".

Il rapporto con il cibo può condurre anche a vere e proprie forme di rielaborazione dei significati degli alimenti del paese di origine tramite il contatto con altre culture. Tale processo si declina in modi diversi, intervenendo sia sui prodotti che sulle modalità di consumo. Così, uno studente palestinese di 21 anni, in Italia fin da bambino, presenta l'immagine dei falafel (pietanza mediorientale costituita da polpette di legumi) accompagnati da patatine fritte descrivendoli come "una sorta di fish and chips" da poter ingolosire con "salsa di ketchup". Ne deriva una contaminazione di un piatto tradizionale (i falafel) con modi di consumo da fast food, globalizzati, pratica che, per stessa ammissione dell'intervistato, non sarebbe praticabile nel paese d'origine: "non credo l'avrei fatto in Palestina". Dal canto suo, invece, un intervistato marocchino di 24 anni - in Italia con i genitori dall'età di 3 anni - racconta di aver ormai preso l'abitudine di mangiare cous cous con la forchetta, specie da quando ha iniziato le scuole in Italia e si è ritrovato, nel quotidiano, a tavola assieme a bambini di altre origini. Nel privato, in famiglia, continua però a mangiarlo con le mani, come la tradizione del paese d'origine vorrebbe ("è un modo per dire grazie a Dio per il cibo ricevuto"). $\mathrm{La}$ negoziazione delle norme alimentari in contesto migratorio conduce quindi all'elaborazione di tecniche di adattamento situate, in cui i migranti si 
ritrovano a dover conciliare orizzonti e pratiche culturali in base alle interazioni e ai loro protagonisti.

\section{Sud vs Nord? Etnografia virtuale del "pacco da giù"}

In ultimo, analizziamo il fenomeno del "pacco da giù", l'invio periodico di provviste alimentari da parte dei familiari di studenti e studentesse universitarie protagoniste di una migrazione scolastica interna (Panichella, 2014; Scavarda, 2012) dalle regioni del Sud Italia (in particolare Puglia, Calabria e Campania) in direzione dei grandi Atenei del Nord Italia ${ }^{5}$. Tale pratica ha contribuito alla costruzione di una specifica identità migrante, quella del "terrone fuori sede" ${ }^{\prime \prime}$, che unisce il rimando alla propria origine geografica (essere "terroni", provenienti dal Meridione italiano) e alla propria condizione di migranti intellettuali (essere "studenti fuori sede"). Con un processo di rovesciamento dello stigma, l'etichetta "terrone" diviene oggetto di riappropriazione e di risignificazione. Il gusto per il "proprio" cibo e una sorta di avversione per quello altrui sono ostentati come strumento per segnare il confine tra gruppi sociali (Niola, 2015).

Nel nostro caso di studio, tali processi sono supportati e rafforzati dalla possibilità di rendere visibili, far circolare e condividere rappresentazioni del cibo tramite social network, come Facebook, Instagram e Youtube, che ospitano profili individuali o di gruppo e canali tematici dedicati ad una meridionalità reinventata e mitizzata, spesso in chiave umoristica e autoironica. Queste piattaforme non offrono semplicemente uno spazio virtuale per condividere l'esperienza migratoria attraverso il cibo e il suo ruolo di integrazione lontano da casa, ma operano come vere e proprie comunità di pratiche anche immaginate (Anderson, 1983) in cui i partecipanti hanno ruolo attivo tramite post e commenti in riferimento ai contenuti pubblicati. L'etnografia virtuale ha quindi indagato i materiali visuali e testuali (immagini, video, didascalie e commenti) caricati e condivisi su alcuni social media dedicati alla figura del "Terrone Fuori Sede" e al suo rapporto col cibo.

5. Questo paragrafo si basa sull'etnografia virtuale realizzata da Cina Tanzarella - che ringraziamo per la concessione dell'uso dei materiali - per la sua tesi di laurea triennale "Terroni Fuori Sede. Pratiche di costruzione identitaria fra i migranti italiani oggi", discussa nel settembre 2018 sotto la supervisione di una delle autrici.

6. La figura del "terrone fuori sede" è descritta come apparentemente neutra dal punto di vista del genere perché definita da una relazione con la madre che colloca il figlio (specie se maschio, ma qualcosa di simile avviene anche per le figlie femmine) nella duplice posizione subordinata di giovane inesperto in cucina e privo delle reti di protezione alimentare del paese d'origine. 
Nella retorica della pagina Facebook de Il Terrone Fuori Sede (TFS), il cibo è inteso in quanto strumento di mantenimento di un legame, materiale e simbolico, con la terra di origine. Ciò appare evidente nel decalogo parodistico delle caratteristiche per riconoscere il vero TFS che "non compra l'olio, lo sale da giù"; riceve con cadenza regolare dei pacchi pieni di scorte di cibo premurosamente preparate in casa; ha una mamma "ossessionata dal cibo e dalla domanda "hai mangiato?"'" Quest'ultimo punto rivela la dimensione di genere del dono di cibo (Lupton, 1999) e il suo valore simbolico che, in base alla quantità di amore e tempo investita nella sua preparazione, trasforma il legame affettivo in arte e cultura, come attesta questo post: "Si chiama boccaccio perché dentro c'è poesia"7.

Il "pacco da giù" agisce in modo performativo nel costruire l'identità del TFS, distinta dalla figura del "polentone", attraverso l'esibizione di specifici generi alimentari: se il canale tematico di Youtube Casa Surace dedica numerosi video all'argomento (per esempio, "Come preparare il pacco da giư", "Quando arriva il pacco da giù", "Quando finisci il pacco da giù" $"$ ), i profili Instagram e Facebook del TFS ospitano immagini di piatti tipici accompagnati da descrizioni nostalgiche o foto di "pacchi da giù" esibiti come tesori, ricolmi di barattoli di verdure sottolio e conserve di ogni tipo, alla cui apertura si sprigionano suoni ed odori ritenuti inafferrabili per i "polentoni".

Le distinzioni fra "terroni" e "polentoni" si giocano anche sul piano del sistema metrico utilizzato per misurare il cibo: si vedano, per esempio, i video di Casa Surace dal titolo "Le unità di misura del Terrone"" "La vita con un Terrone $v s$ senza un Terrone"12). La rappresentazione autoironica del "cibo di giù" è giocata sul suo eccesso: "Mangiare certe cose (e mangiarle preparate in un certo modo) non è [quindi] più solo il frutto di un'abitudine o di una scelta. È il segno di un'identità sociale" (Montanari, 1993: 107-108).

La celebrazione ritualizzata della condivisione dei prodotti del "pacco da giù" con i compagni di esilio costituisce, in questa prospettiva, l'ultimo momento del processo di creazione della meridionalità migrante, che ricostruisce un più esplicito senso del noi attraverso l'organizzazione di maratone alimentari con altri TFS (si veda il video "Il frigo: Nord $v s$ Sud") ${ }^{13}$. La con-

7. I boccacci sono contenitori in vetro per la conservazione del cibo. Si veda il link https://www.instagram.com/p/BhyfrYzlTrq/ .

8. https://www.youtube.com/watch?v=Etlb-7L4IFs

9. https://www.youtube.com/watch?v=lHIIhP9SCaQ

10. https://www youtube.com/watch?v=exwgk7Thj4s

11. https://www.youtube.com/watch?v=KP Y014pdKo

12. https://www.youtube.com/watch?v=oKquOr3ZJpQ

13. https://www.youtube.com/watch?v=tD3BT4QvDx8 
divisione del cibo passa anche attraverso la dimensione virtuale della rappresentazione dei cibi ricevuti, offerti allo sguardo e al vissuto emotivo social. La pratica del mangiare insieme, reale o virtuale, costituisce così uno strumento di costruzione di un nuovo rapporto tra popolazioni minoritarie e maggioritarie: stavolta sullo stesso territorio nazionale, distinguendo "noi" (chi migra dal Sud) e "loro" ("i polentoni").

La rappresentazione celebrativa della meridionalità sui media digitali analizzati è collocata all'interno di un registro (auto)ironico. Il ridere con qualcuno e di qualcuno, anche di se stessi, rappresenta uno strumento di costruzione di confini, appartenenze e complicità così come di differenze e distinzioni, che va nella direzione dell'ibridazione: permette di giocare con le rappresentazioni stereotipate, riappropriandosi di alcuni tratti (ad esempio, la connessione tra cibo preparato nella famiglia d'origine, veicolo di ricordi, emozioni e relazioni) mentre ci si distanzia discorsivamente da altri (come la figura della madre onnipresente) ${ }^{14}$.

\section{Osservazioni conclusive}

I tre casi analizzati mostrano come l'alimentazione illumini versanti specifici dei percorsi migratori, raccontando come e perché le popolazioni migranti stabiliscono una relazione con la comunità d'accoglienza in base a ciò che mangiano; se, e in cosa, chi migra si consideri "diverso" da chi accoglie; come e perché questa diversità crei talvolta inaspettate corrispondenze, talaltra rinnovi le distanze. Se la letteratura sociologica sull'alimentazione si è soprattutto interessata alle dinamiche di auto ed etero-etichettamento che accompagnano le diete scelte da gruppi e individui (le scelte vegetariane e vegane per esempio, cfr. Cole e Morgan, 2011), il nostro contributo ha esplorato tali logiche focalizzandosi su pratiche alimentari "ordinarie", non necessariamente alternative, che assumono significati peculiari proprio perché immerse nel contesto migratorio.

In particolare, il ricorso ad approcci qualitativi ha fatto emergere specifiche forme di creatività culturale nelle migrazioni (Saint-Blancat, 2004) in cui le appartenenze d'origine (religiose e culturali) sono ripensate e rielaborate attraverso i piatti e le preparazioni culinarie. I casi di studio presi in esame mostrano l'accezione più ampia dei significati del cibo: non solo strumento di nutrizione, ma anche - e soprattutto - oggetto culturale di cui gli individui possono servirsi per costruire ruoli (Fischler, 1992) e relazioni sociali create

14. Per un approfondimento del ricorso al linguaggio umoristico e ironico come forma di ibridazione attraverso il distanziamento discorsivo, cfr. Bridges e Pascoe (2014). 
e sostenute dai consumi (Franchi, 2009). La specificità delle analisi qualitative sviluppate, inoltre, ci ha portato a riconoscere come l'alimentazione in migrazione sia uno spazio privilegiato per rivendicare una identità culturale d'origine, da ricostruire in modo nostalgico o da riaffermare in quanto tratto distintivo rispetto a chi accoglie. Ne emerge una definizione di confini simbolici che opera come una dialettica di opposizione tra "voi" e "noi" in cui l'alimentazione può finanche trasformarsi in strumento che neutralizza e ribalta lo stigma (Goffman, 1983). Allo stesso tempo, il cibo può aprire varchi di scambio e comunicazione, trasformandosi in un terreno di incontro e di ibridazione di tradizioni diverse, attraverso la (de)costruzione di confini continuamente rinegoziati, e contribuendo ad attenuare quella stessa opposizione "voi" vs "noi", pur non annullandola del tutto. La rivendicazione orgogliosa di una identità migrante passa allora attraverso le tappe materiali degli itinerari alimentari (dalla scelta dei prodotti, alla preparazione, sino al loro consumo), e trova oggi nuove arene espressive grazie al ruolo crescente dei social media che diventano spazio di rappresentazione di sé, in cui la messa in scena del cibo e del suo radicamento culturale contribuisce alla costruzione di una comunità immaginata migrante e alimentare.

\section{Bibliografia}

Anderson B. (1983). Imagined communities: reflections on the origin and spread of nationalism, Londra: Verso.

Bojorquez I., Rentería D., Unikel C. (2014). Trajectories of dietary change and the social context of migration: a qualitative study. Appetite, 81: 93-101. https://doi.org/10.1016/j.appet.2014.06.005.

Bridges T. e Pascoe C.J. (2014). Hybrid masculinities: New directions in the sociology of men and masculinities. Sociology Compass, 8, 3, 246-258. https://doi.org/10.1111/soc4.12134.

Cardano M. (2020) Argomenti per la ricerca qualitativa. Bologna, Il Mulino.

Cole M., Morgan K. (2011). Vegaphobia: derogatory discourses of veganism and the reproduction of speciesism in UK national newspapers. The British Journal of Sociology, 62, 1: 134-153, https://doi.org/10.1111/j.1468-4446.2010.01348.x.

Colombo E. e Semi G., a cura di (2007). Multiculturalismo quotidiano. Le pratiche della differenza Milano: FrancoAngeli.

Cottino G. (2020). La patata. Contese alimentari tra migrazioni e mobilità nelle valli alpine occidentali. In: Ascari P., a cura di. Oggetti contesi. Le cose nella migrazione (pp. 143-159). Milano: Mimesis.

Crenn C. (2020). La ronde des mondes. Migrants et aliments en circulation en Afrique et en Europe. Parigi: Karthala.

Douglas M. (1973). Food in the social order. London: Routledge.

Fassin D. (2009). Les économies morales revisitées. Annales HSS, 6: 1237-1266. 
Ferrero Camoletto R. e Scavarda A. (2020). Il cibo come specchio: la costruzione delle maschilità tra adolescenti e giovani uomini. AG About Gender-Rivista internazionale di studi di genere, 9, 17: 97-141; https://doi.org/10.15167/22795057/AG2020.9.17.1161.

Fidolini V. (2019). Sorvegliare la salute? Uomini e costruzioni di genere attraverso le pratiche alimentari, Rassegna Italiana di Sociologia, LX, 2: 229-330.

Fidolini V. (2016). Costruzioni della maschilità nella transizione all'età adulta. Modelli egemoni e scripts sessuali tra i giovani marocchini in Italia e in Francia. Polis. Ricerche e studi su società e politica in Italia, 30, 1: 87-122.

Fischler C. (1992). L'onnivoro. Il piacere di mangiare nella storia e nella scienza, Milano: Arnoldo Mondadori.

Franchi M. (2009). Il cibo flessibile. Nuovi comportamenti di consumo. Roma: Carocci.

Gabaccia D.R. (1998). We are what we eat: ethnic food and the making of Americans. Harvard: Harvard University Press.

Goffman E. (1983). Stigma. L'identità negate. Milano: Giuffré

Harper D. (2012). Visual sociology. London: Routledge.

Janowski M. (2012). Introduction: Consuming Memories of Home in Constructing the Present and Imagining the Future. Food and Foodways, 20: 3-4; https://doi.org/10.1080/07409710.2012.715960.

Lamont M., Pendergrass S. e Pachucki M. (2001). Symbolic Boundaries. International Encyclopedia of the Social and Behavioral Sciences, 23: 15341-15347.

Librandi F. (2005). Il gusto e i ritmi della memoria. In: La Camera C., a cura di, Praxis. Voci e memorie della cultura popolare (pp. 68-82). Reggio Calabria: Baruffa.

Lin H.M., Pang C.L. e Liao D.C. (2020). Home food making, belonging, and identity negotiation in Belgian Taiwanese immigrant women's everyday food practices. Journal of Ethnic Foods, 7, 1: 1-18.

Lupton D. (1999). L'anima nel piatto. Bologna: il Mulino.

Middha B. (2018). Everyday digital engagements: using food selfies on Facebook to explore eating practices. Communication Research and Practice, 4, 3: 291-306: https://doi.org/10.1080/22041451.2018.1476796.

Montanari M. (1993). La fame e l'abbondanza. Roma-Bari: Laterza.

Niola M. (2015). Homo dieteticus. Viaggio nelle tribù alimentari. Bologna: il Mulino.

Panichella N. (2014). Meridionali al Nord. Migrazioni interne e società italiana dal dopoguerra ad oggi. Bologna: il Mulino.

Poulain J.-P. (2008). Alimentazione, cultura e società. Bologna: il Mulino.

Raman P. (2011). Me in Place, and the Place in Me. A Migrant's Tale of Food, Home and Belonging. Food, Culture and Society, 14, 2: 165-180; https://doi.org/10.2752/175174411X12893984828674.

Ray K. (2004). The Migrant's Table: Meals and Memories in Bengali-American Households. Philadelphia: Temple University Press. 
Saint-Blancat C. (2004). La transmission de l'islam auprès des nouvelles générations de la diaspora. Social Compass, 51, 2: 235-247, https://doi.org/10.1177/0037768604043009.

Scavarda A. (2012). La generazione immobile ha ripreso a spostarsi: il caso degli studenti meridionali a Torino. Meridiana, 75: 131-148.

Soriano-Ayala E., Cala V. C., Ruiz-Salvador D. (2020). Identification of Cultural and Transcultural Health Assets among Moroccan, Romanian and Spanish Adolescents through Photovoice. Journal of Immigrant and Minority Health, 22, 2: 255-265; https://doi.org/10.1007/s10903-019-00934-1.

Stagi L. (2016). Food Porn. L'ossessione per il cibo in TV e nei social media. Milano: Egea.

Taylor N. e Keating M. (2018). Contemporary food imagery: food porn and other visual trends. Communication Research and Practice, 4, 3: 307-323; https://doi.org/10.1080/22041451.2018.1482190.

Terragni L. e Roos G. (2018). People Moving with Food from and to Northern Europe: Food, Migration and Multiculturalism. Anthropology of food, 12; https://doi.org/10.4000/aof.9123.

Teti V. (2020). Nostalgia: Antropologia di un sentimento del presente. Bologna: Marietti 182. 